Available at: http://publications.ictp.it

IC $/ 2007 / 118$

United Nations Educational, Scientific and Cultural Organization

and

International Atomic Energy Agency

THE ABDUS SALAM INTERNATIONAL CENTRE FOR THEORETICAL PHYSICS

\title{
A SYMPLECTIC GENERALIZATION OF THE PERADZYŃSKI HELICITY THEOREM AND SOME APPLICATIONS
}

\author{
Anatoliy K. Prykarpatsky1 \\ The AGH University of Science and Technology, Kraków 30-059, Poland, \\ The IAPMM of the National Academy of Sciences, Lviv, Ukraine \\ and \\ The Abdus Salam International Centre for Theoretical Physics, Trieste, Italy, \\ Nikolai N. Bogoliubov (Jr.)2 \\ V.A. Steklov Mathematical Institute of RAN, Moscow, Russian Federation \\ and \\ The Abdus Salam International Centre for Theoretical Physics, Trieste, Italy \\ and \\ Jolanta Golenia ${ }^{3}$ \\ Department of Applied Mathematics at the AGH University of Science and Technology, \\ Kraków 30-059, Poland.
}

\begin{abstract}
Symplectic and symmetry analysis for studying MHD superfluid flows is devised, a new version of the Z. Peradzyński 8 helicity theorem based on differential-geometric and group-theoretical methods is derived. Having reanalyzed the Peradzyński helicity theorem within the modern symplectic theory of differential-geometric structures on manifolds, a new unified proof and a new generalization of this theorem for the case of compressible MHD superfluid flow are proposed. As a by-product, a sequence of nontrivial helicity type local and global conservation laws for the case of incompressible superfluid flow, playing a crucial role for studying the stability problem under suitable boundary conditions, is constructed.
\end{abstract}

\section{MIRAMARE - TRIESTE}

December 2007

\footnotetext{
${ }^{1}$ pryk.anat@ua.fm, prykanat@cybergal.com

2 nikolai_bogolubov@hotmail.com

3 goljols@tlen.pl
} 


\section{Introduction}

Long ago it was stated [7, 5] that quantum vortices in superfluid helium can be studied either as open lines with their ends terminating on free surfaces of walls of the container or as closed curves. Nowadays the closed vortices are treated as topological objects equivalent to circles. The existence of structures such as knotted and linked vertex lines in the turbulent phase is almost obvious [12] and forces researchers to develop new mathematical tools for their detailed investigation. In this proposed direction it was proved by Z. Peradzyński [8] a new version of the Helicity theorem, based on some differential-geometric methods, applied to the description of the collective motion in the in-compressible superfluid. The Peradzyński helicity theorem describes in a unique way, both the superfluid equations and the related helicity invariants, which are, in the conservative case, very important for studying the topological structure of vortices.

Having reanalyzed the Peradzyński helicity theorem within the modern symplectic theory of differential-geometric structures on manifolds, we propose a new unified proof and give a magneto-hydrodynamic generalization of this theorem for the case of an incompressible superfluid flow. As a by-product, in the conservative case we construct a sequence of nontrivial helicity type conservation laws, which play a crucial role for studying the stability problem of superfluid under suitable boundary conditions.

\section{$2 \quad$ Symplectic and symmetry analysis}

We consider a quasi-neutral superfluid contained in a domain $M \subset \mathbb{R}^{3}$ and interacting with a "frozen" magnetic field $B: M \longrightarrow \mathbb{E}^{3}$, where $\mathbb{E}^{3}:=\left(\mathbb{R}^{3},<., .>\right)$ is the standard threedimensional Euclidean vector space with the scalar $<., .>$ and vector " $\times$ " products. The magnetic field is considered to be source-less and satisfying the condition $B=\nabla \times A$, where $A: M \longrightarrow \mathbb{E}^{3}$ is some magnetic field potential. The corresponding electric field $E: M \longrightarrow \mathbb{E}^{3}$, related with the magnetic potential, satisfies the necessary superconductivity conditions

$$
E+u \times B=0, \quad \partial E / \partial t=\nabla \times B,
$$

where $u: M \longrightarrow T(M)$ is the superfluid velocity.

Let $\partial M$ denote the boundary of the domain $M$. The following boundary conditions $\left.\langle n, u\rangle\right|_{\partial M}=$ 0 and $\left.\langle n, B\rangle\right|_{\partial M}=0$ are imposed on the superfluid flow, where $n \in T^{*}(M)$ is the vector normal to the boundary $\partial M$, considered to be almost everywhere smooth.

Then in adiabatic magneto-hydrodynamics (MHD) quasi-neutral superfluid motion can be described, using (2.1), by the following system of evolution equations:

$$
\begin{gathered}
\partial u / \partial t=-\langle u, \nabla\rangle u-\rho^{-1} \nabla P+\rho^{-1}(\nabla \times B) \times B, \\
\partial \rho / \partial t=-\langle\nabla, \rho u\rangle, \quad \partial \eta / \partial t=-\langle u, \nabla \eta\rangle, \quad \partial B / \partial t=\nabla \times(u \times B),
\end{gathered}
$$

where $\rho: M \longrightarrow \mathbb{R}_{+}$is the superfluid density, $P: M \longrightarrow \mathbb{E}^{3}$ is the internal pressure and $\eta: M \longrightarrow \mathbb{R}$ is the specific superfluid entropy. The latter is related to the internal MHD 
superfluid specific energy function $e=e(\rho, \eta)$ owing to the first thermodynamic law:

$$
T d \eta=d e(\rho, \eta)-P \rho^{-2} d \rho
$$

where $T=T(\rho, \eta)$ is the internal absolute temperature in the superfluid. The system of evolution equations (2.2) conserves the total energy

$$
H:=\int_{M}\left[\frac{1}{2 \rho}|\mu|^{2}+\rho e(\rho, \eta)+\frac{1}{2}|B|^{2}\right] d^{3} x
$$

called the Hamiltonian, since the dynamical system (2.2) is a Hamiltonian system on the functional manifold $\mathcal{M}:=C^{\infty}\left(M ; T^{*}(M) \times \mathbb{R}^{2} \times \mathbb{E}^{3}\right)$ with respect to the following [4] Poisson bracket:

$$
\begin{aligned}
\{f, g\}: & =\int_{M}\left\{\left\langle\mu,\left[\frac{\delta f}{\delta \mu}, \frac{\delta g}{\delta \mu}\right]_{c}\right\rangle+\rho\left(\left\langle\frac{\delta g}{\delta \mu}, \nabla \frac{\delta f}{\delta \rho}\right\rangle-\left\langle\frac{\delta f}{\delta \mu}, \nabla \frac{\delta g}{\delta \rho}\right\rangle\right)\right. \\
& +\eta\left\langle\nabla,\left(\frac{\delta g}{\delta \mu} \frac{\delta f}{\delta \eta}-\frac{\delta f}{\delta \mu} \frac{\delta g}{\delta \eta}\right)\right\rangle+\left\langle B,\left[\frac{\delta g}{\delta \mu}, \frac{\delta f}{\delta B}\right]_{c}\right\rangle \\
& \left.+\left\langle\frac{\delta f}{\delta B},\langle B, \nabla\rangle \frac{\delta g}{\delta \mu}\right\rangle-\left\langle\frac{\delta g}{\delta B},\langle B, \nabla\rangle \frac{\delta f}{\delta \mu}\right\rangle\right\} d x
\end{aligned}
$$

where we denoted by $\mu:=\rho u \in T^{*}(M)$ the specific momentum of the superfluid motion and by $[.,]_{c}$ the canonical Lie bracket of variational gradient vector fields:

$$
\left[\frac{\delta f}{\delta \mu}, \frac{\delta g}{\delta \mu}\right]_{c}:=\left\langle\frac{\delta f}{\delta \mu}, \nabla\right\rangle \frac{\delta g}{\delta \mu}-\left\langle\frac{\delta g}{\delta \mu}, \nabla\right\rangle \frac{\delta f}{\delta \mu}
$$

for any smooth functionals $f, g \in \mathcal{D}(M)$ on the functional space $\mathcal{M}$. Moreover, as it was stated in [4], the Poisson bracket (2.5) is, in reality, the canonical Lie-Poisson bracket on the dual space to the Lie algebra $\mathcal{G}$ of the semidirect product of vector fields on $M$ and the direct sum of functions, densities and differential one-forms on $M$. Namely, the specific momentum $\mu=\rho u \in T^{*}(M)$ is dual to vector fields, $\rho$ is dual to functions, $\eta$ is dual to densities and $B$ is dual to the space of two-forms on $M$. Thus, the set of evolution equations (2.2) can be equivalently re-written as follows:

$$
\begin{aligned}
\partial u / \partial t=\{H, u\}, & \partial \rho / \partial t=\{H, \rho\}, \\
\partial \eta / \partial t=\{H, \eta\}, & \partial B / \partial t=\{H, B\} .
\end{aligned}
$$

The Poisson bracket (2.5) can be re-written for any $f, g \in \mathcal{D}(M)$ as

$$
\{f, g\}=(D f, \vartheta D g)
$$

with $D f:=\left(\frac{\delta f}{\delta \mu}, \frac{\delta f}{\delta \rho}, \frac{\delta f}{\delta \eta}, \frac{\delta f}{\delta B}\right)^{\top} \in T^{*}(\mathcal{M})$ and $\vartheta: T^{*}(\mathcal{M}) \longrightarrow T(\mathcal{M})$, being the corresponding (modulo the Casimir functionals of bracket (2.5)) invertible [3] co-symplectic operator, satisfying the standard [10, 2] properties

$$
\vartheta^{*}=-\vartheta, \quad \delta\left(\delta w, \wedge \vartheta^{-1} \delta w\right)=0
$$

where the differential variation complex condition $\delta^{2}=0$ is assumed, the differential variation vector $\delta w:=(\delta \mu, \delta \rho, \delta \eta, \delta B)^{\top} \in T^{*}(\mathcal{M})$ and the sign "*” denotes the conjugate mapping with 
respect to the standard bi-linear convolution $(.,$.$) of two spaces T^{*}(\mathcal{M})$ and $T(\mathcal{M})$. Note here that the second condition of (2.9) is equivalent [2, 10, to the fact that the Poisson bracket (2.5) satisfies the Jacobi commutation condition. Thus, one can define the closed generalized variational differential two-form on $\mathcal{M}$

$$
\omega^{(2)}:=\left(\delta w, \wedge \vartheta^{-1} \delta w\right),
$$

being a symplectic structure on the functional factor manifold $\mathcal{M}$ (modulo the Casimir functionals of bracket (2.5) ).

Denote now a subgroup $\mathcal{D}_{t}(M)=\left\{\varphi_{t}: M \rightarrow M\right\}$ of the diffeomorphism group Dif $f_{+} M$, consisting of invertible transformations $\varphi_{t}: M \rightarrow M$, generated by MHD superfluid evolution equations (2.2). This means, by definition, that

$$
d \varphi_{t}(x) / d t:=u\left(\varphi_{t}(x)\right)
$$

for all $x \in M$ and suitable $t \in \mathbb{R}$, for which solutions to (2.2) exist and are unique. The symplectic structure (2.10) is invariant with respect to the induced mapping of diffeomorphisms $\hat{\varphi}_{t}: \mathcal{M} \rightarrow \mathcal{M}$ on the functional manifold $\mathcal{M}$, that is

$$
\hat{\varphi}_{t, *} \omega^{(2)}=\omega^{(2)}
$$

for suitable $t \in \mathbb{R}$. Then the corresponding diffeomorphism subgroup $\hat{\mathcal{D}}_{t}(\mathcal{M}):=\left\{\hat{\varphi}_{t}: \mathcal{M} \rightarrow \mathcal{M}\right\}$ satisfies the evolution equation

$$
d \hat{\varphi}_{t}(w) / d t:=K_{H}\left(\hat{\varphi}_{t}(w)\right)
$$

for any $w \in \mathcal{M}$ and the same suitable $t \in \mathbb{R}$, where the vector field $K_{H}: \mathcal{M} \longrightarrow T(\mathcal{M})$ coincides with the system of MHD evolution equations (2.2). This fact easily follows from the standard [2] differential-geometric considerations related to equality (2.12). Really, from (2.12) one obtains that

$$
0=\frac{d}{d t} \hat{\varphi}_{t, *} \omega^{(2)}:=L_{K_{H}} \omega^{(2)}=\left(i_{K_{H}} \delta+\delta i_{K_{H}}\right) \omega^{(2)}=\delta i_{K_{H}} \omega^{(2)}
$$

for all these suitable $t \in \mathbb{R}$, where we denoted by $L_{K_{H}}$ the standard Lie derivative with respect to the vector field $K_{H}$ on $\mathcal{M}$ and used the corresponding Cartan formula $L_{K_{H}}=i_{K_{H}} \delta+\delta i_{K_{H}}$. Now, owing to the Hamiltonian equations (2.7), the equality $i_{K_{H}} \omega^{(2)}=-\delta H$ holds, and since $\delta^{2}=0$ the invariance property (2.12) is stated.

As the properties of equations (2.2) on the manifold $\mathcal{M}$ are completely determined by the diffeomorphism subgroup $\mathcal{D}_{t}(M) \subset D$ if $f_{+}(M)$, we will reformulate further the set of equations (2.2) making use of the suitable invariant properties on the manifold $M$. First, observe that the mass conservation law of our superfluid flow is equivalent to the equality

$$
\frac{d}{d t} \int_{D_{t}} \rho d^{3} x=0
$$

for any domain $D_{t} \subset M$ moving together with chosen inside particles. It is an easy calculation to rewrite (2.15) in the following equivalent form:

$$
\int_{D_{t}}\left(\partial / \partial t+L_{u}\right)\left(\rho d^{3} x\right)=0
$$


for all domains $D_{t} \subset M$ and suitable $t \in \mathbb{R}$, where as above, we denoted by $L_{u}=i_{u} d+d i_{u}$ the Lie derivative along the vector field $u: M \longrightarrow T(M)$ on $M$ in the Cartan form.

As a result of (2.16) one obtains the following local differential-geometric relationship:

$$
\left(\partial / \partial t+L_{u}\right)\left(\rho d^{3} x\right)=0
$$

Since the evolution of our superfluid is locally adiabatic, the following equality

$$
\left(\partial / \partial t+L_{u}\right) \eta=0
$$

is obvious, meaning only that $d \eta / d t=0$ for all suitable $t \in \mathbb{R}$.

Now take the momentum conservation law in the integral Amper-Newton form

$$
\frac{d}{d t} \int_{D_{t}} \rho u d^{3} x+\int_{S_{t}=\partial D_{t}} P d S_{t}-\int_{D_{t}}(j \times B) d^{3} x=0,
$$

where $d S_{t}$ is the corresponding oriented surface measure on the boundary $S_{t}:=\partial D_{t}$ of a domain $D_{t} \subset M, P: M \longrightarrow \mathbb{R}$ is the internal pressure and $j: M \longrightarrow \mathbb{E}^{3}$ is the corresponding induced current density in the MHD superfluid under the superconductivity condition. The latter means that, owing to neutrality of the superfluid, the induction condition

$$
\nabla \times B+j=0
$$

holds. Then from (2.19) and (2.20) one easily obtains the infinitesimal form of the evolution for the velocity vector $u: M \longrightarrow T(M)$ :

$$
\left(\partial / \partial t+L_{K_{H}}\right) u=-\rho^{-1} \nabla P+\rho^{-1}(\nabla \times B) \times B
$$

coinciding, evidently, with the first equation of system (2.2).

Consider now at each moment of $t \in \mathbb{R}$ the subgroup of diffeomorphisms $\mathcal{D}_{\tau}=\left\{\psi_{\tau}: M \rightarrow M\right\} \subset$ $\operatorname{Diff}(M)$, generated by the following vector field $v: M \longrightarrow T(M)$ on $M$ :

$$
d \psi_{\tau}(x) / d \tau:=v\left(\psi_{\tau}(x)\right)=\rho^{-1} B\left(\psi_{\tau}(x)\right)
$$

defined for a suitable evolution parameters $\tau \in \mathbb{R}$. Since the subgroup $\mathcal{D}_{\tau}$ does not depend explicitly on the evolution parameter $t \in \mathbb{R}$, its action can be interpreted as re-arranging the superfluid particles within any chosen domain $D_{t} \subset M$. Owing now to the commutation property

$$
\left[\partial / \partial t+L_{u}, L_{v}\right]=0
$$

equivalent to commuting subgroup $\mathcal{D}_{t}$ and $\mathcal{D}_{\tau}$ for any suitable $t, \tau \in \mathbb{R}$, from the invariance condition

$$
\partial \rho / \partial \tau=0
$$

we can derive that quantities

$$
\gamma_{n}:=L_{v}^{n} \gamma
$$


for all $n \in \mathbb{Z}_{+}$are invariants of the MHD superfluid flow (2.2), if the density $\gamma \in \Lambda^{3}(M)$ is also an invariant on $M$. Really, we have

$$
\left(\partial / \partial t+L_{u}\right) \gamma_{n}=\left(\partial / \partial t+L_{u}\right) L_{v}^{n} \gamma=L_{v}^{n}\left(\partial / \partial t+L_{u}\right) \gamma=0
$$

since, by definition, we have

$$
\left(\partial / \partial t+L_{u}\right) \gamma=0
$$

Such a density can be found, observing [4] that the superconductivity conditions $E+u \times B=0$, $E=-\partial A / \partial t$ and the last equation of system (2.2) brings about the invariance condition

$$
\left(\partial / \partial t+L_{u}\right) d \alpha^{(1)}=0
$$

where the one-form $\alpha^{(1)} \in \Lambda^{1}(M)$ equals

$$
\alpha^{(1)}:=\langle A, d x\rangle
$$

Moreover, since the differential operations $\partial / \partial t+L_{u}$ and " $d$ " commute [2], one checks that the stronger cohomological condition

$$
\left(\partial / \partial t+L_{u}\right) \alpha^{(1)}=0
$$

holds on $M$, if the time-dependent gauge mapping $A \longrightarrow A+\nabla \psi$, where $\partial \psi / \partial t+L_{u} \psi+\langle u, A\rangle=0$, is applied to the magnetic potential $A: M \longrightarrow \mathbb{E}^{3}$. Now from conditions (2.28) and (2.30) one easily derives that the density

$$
\gamma:=\alpha^{(1)} \wedge d \alpha^{(1)}
$$

satisfies equation (2.27). Thus, it generates, in view of formula (2.25), new conserved quantities, which can be equivalently rewritten as

$$
\tilde{\gamma}_{n}:=\rho L_{v}^{n}\left(\rho^{-1}\langle B, A\rangle\right)=\rho L_{v}^{n}\langle v, A\rangle
$$

for all $n \in \mathbb{Z}_{+}$. Thereby, the following functionals on the functional manifold $\mathcal{M}$

$$
\tilde{H}_{n}:=\int_{M} \tilde{\gamma}_{n} d^{3} x=\int_{M} \rho L_{v}^{n}\left(\rho^{-1}\langle B, A\rangle\right) d^{3} x
$$

for all $n \in \mathbb{Z}_{+}$are invariants of our MHD superfluid dynamical system (2.2). In particular, at $n=0$ we obtain the well-known [4] magnetic helicity invariant

$$
\tilde{H}_{0}=\int_{M}\langle A, \nabla \times A\rangle d^{3} x
$$

which exists independently of boundary conditions, imposed on the MHD superfluid flow equations (2.2).

The result obtained above can be formulated as the following theorem.

Theorem 2.1 The functionals (2.33), where the Lie derivative $L_{v}$ is taken along the magnetic vector field $v=\rho^{-1} B$, are global invariants of the system of compressible MHD superfluid and superconductive equations (2.2). 
Below we proceed to symmetry analysis of the incompressible superfluid dynamical system and construct the related local and global new helicity invariants. The case of superfluid hydrodynamical flows [9] is of great interest for many applications owing to the very nontrivial dynamical properties of so-called vorticity structures, featuring the motion.

\section{The incompressible superfluid: symmetry analysis and conser- vation laws}

Concerning the helicity theorem result of [8], where the kinematic helicity invariant

$$
H_{0}:=\int_{M}\langle u, \nabla \times u\rangle d^{3} x
$$

was derived, making use of differential-geometric tools in Minkowski space in the case of incompressible superfluid at the absent magnetic field $B=0$, we will show below its general dynamical symmetry nature. The governing equations look as follows:

$$
\partial u / \partial t=-\langle u, \nabla\rangle u+\rho^{-1} \nabla P, \quad \partial \rho / \partial t+\langle u, \nabla \rho\rangle=0, \quad\langle\nabla, u\rangle=0,
$$

where the density conservation properties

$$
\left(\partial / \partial t+L_{u}\right) \rho=0, \quad\left(\partial / \partial t+L_{u}\right) d^{3} x=0
$$

hold for all suitable $t \in \mathbb{R}$. Define now the vorticity vector $\xi:=\nabla \times u$ and find from (3.2) that it satisfies the vorticity flow equation

$$
\partial \xi / \partial t=\nabla \times(u \times \xi)
$$

Really, the first equation of (3.2) can be rewritten as

$$
\partial u / \partial t=u \times(\nabla \times u)-\rho^{-1} \nabla P-\frac{1}{2} \nabla|u|^{2} .
$$

Then, applying the operation " $\nabla \times$." to (3.5), one easily obtains the vorticity equation (3.4). Moreover, equation (3.4) can be rewritten in the equivalent form

$$
\partial \xi / \partial t+\langle u, \nabla\rangle \xi=\langle\xi, \nabla\rangle u
$$

which allows a new dynamical symmetry interpretation.

Put, by definition,

$$
\partial x / \partial \tau=v(x, t):=\rho^{-1} \xi,
$$

defining for all $\tau \in \mathbb{R}$ the diffeomorphism subgroup $\mathcal{D}_{\tau} \subset$ Diff $M$ of the manifold $M$. It is easy to check that this subgroup commutes with the previous defined subgroup $\mathcal{D}_{t} \subset \operatorname{Diff} M$, since the following condition

$$
\left(\partial / \partial t+L_{u}\right) v=L_{v} u
$$


holds for all $t, \tau \in \mathbb{R}$, exactly coinciding with relationship (3.6). The condition (3.8) means that the commutation property

$$
\left[\partial / \partial t+L_{u}, L_{v}\right]=0
$$

similar to (2.23), holds.

Now we can make use of the invariants generation technique, described above in the case of the superfluid equations (2.2). For this we need to construct a source density invariant $\gamma \in \Lambda^{3}(M)$ of equations (2.26) and construct successively a hierarchy of additional invariants as

$$
\gamma_{n}:=L_{v}^{n} \gamma
$$

for all $n \in \mathbb{Z}_{+}$.

Put, by definition, $\beta^{(1)} \in \Lambda^{1}(M)$ as the one-form

$$
\beta^{(1)}:=\langle u, d x\rangle
$$

and find that

$$
\left(\partial / \partial t+L_{u}\right) \beta^{(1)}=-\rho^{-1} d P+\frac{1}{2} d|u|^{2}=d\left(\rho^{-1} P+\frac{1}{2}|u|^{2}\right) .
$$

The differential two-form $d \beta^{(1)} \in \Lambda^{2}(M)$ satisfies the condition

$$
\left(\partial / \partial t+L_{u}\right) d \beta^{(1)}=d^{2}\left(\rho^{-1} P+\frac{1}{2}|u|^{2}\right)=0
$$

owing to the identity $d^{2}=0$. Then the differential density three-form $\gamma:=\beta^{(1)} \wedge d \beta^{(1)} \in \Lambda^{3}(M)$ satisfies, owing to (3.12) and (3.13), the condition

$$
\begin{gathered}
\left(\partial / \partial t+L_{u}\right) \gamma=\left(\partial / \partial t+L_{u}\right)\left(\beta^{(1)} \wedge d \beta^{(1)}\right) \\
=d\left(\rho^{-1} P+\frac{1}{2}|u|^{2}\right) \wedge d \beta^{(1)}=d\left(\left(\rho^{-1} P+\frac{1}{2}|u|^{2}\right) d \beta^{(1)}\right) .
\end{gathered}
$$

By integration of (3.14) over the whole manifold $M$ we obtain, based on the Stokes theorem, the expression

$$
\begin{gathered}
\frac{d}{d t} \int_{M} \beta^{(1)} \wedge d \beta^{(1)}=\frac{d}{d t} \int_{M}(u \times(\nabla \times u)) d^{3} x=\frac{d}{d t} \int_{M}(u \times \xi) d^{3} x \\
=\oint_{\partial M}\left(\rho^{-1} P+\frac{1}{2}|u|^{2}\right)\langle d u, \wedge d x\rangle=0,
\end{gathered}
$$

if the boundary conditions $\langle u, n\rangle=0$ and $\left.\xi\right|_{\partial M}=0$ are imposed on the superfluid vorticity flow. Really, the surface measure $\langle d u, \wedge d x\rangle$ on the boundary $\partial M$ can be equivalently represented as

$$
\langle d u, \wedge d x\rangle=\langle\langle d x, \nabla\rangle u, \wedge d x\rangle=\langle\nabla \times u, d S\rangle=\langle\xi, d S\rangle,
$$

where $d S$ is the standard oriented Euclidian surface measure on $\partial M$. Since the vorticity vector $\left.\xi\right|_{\partial M}=0$, the result (3.15) follows automatically. 
Assume now that the vorticity vector $\xi=\nabla \times u$ satisfies the additional constraints $\left.L_{v}^{n} \xi\right|_{\partial M}=0$ for $n \in \mathbb{Z}_{+}$. Then we obtain from (3.16) and (2.23) that

$$
\begin{gathered}
\frac{d}{d t} \int_{M} L_{v}^{n} \gamma=\frac{d}{d t} \int_{M} L_{v}^{n} \gamma d^{3} x=\int_{M} L_{v}^{n}\left(\partial / \partial t+L_{u}\right) \gamma \\
=\int_{M} L_{v}^{n} d\left(\rho^{-1} P+\frac{1}{2}|u|^{2}\right) d \beta^{(1)}=\int_{M} d L_{v}^{n}\left(\left(\rho^{-1} P+\frac{1}{2}|u|^{2}\right) d \beta^{(1)}\right) \\
=\int_{M} d L_{v}^{n}\left(\left(\rho^{-1} P+\frac{1}{2}|u|^{2}\right)\langle d u, \wedge d x\rangle\right)=\int_{M} d L_{v}^{n}\left(\left(\rho^{-1} P+\frac{1}{2}|u|^{2}\right)\langle\xi, d S\rangle\right) \\
=\int_{\partial M} L_{v}^{n}\left(\left(\rho^{-1} P+\frac{1}{2}|u|^{2}\right)\langle\xi, d S\rangle\right)=\int_{\partial M} \sum_{k=0}^{n} C_{n}^{k}\left\langle L_{v}^{k} \xi, L_{v}^{n-k}\left(\left(\rho^{-1} P+\frac{1}{2}|u|^{2}\right) d S\right)\right\rangle=0,
\end{gathered}
$$

bringing about the generalized helicity invariants

$$
H_{n}:=\int_{M} \rho L_{v}^{n}(u \times \xi) d^{3} x
$$

for all $n \in \mathbb{Z}_{+}$. Notice here that all of the constraints imposed above on the vorticity vector $\xi=\nabla \times u$ will be automatically satisfied, if the condition supp $\xi \cap \partial M=\emptyset$ holds. The result obtained can be formulated as the following theorem.

Theorem 3.1 Assume that an incompressible superfluid, governed by the set of equations (3.2) in a domain $M \subset \mathbb{E}^{3}$, possesses the vorticity vector $\xi=\nabla \times u$, which satisfies the boundary constraints $\left.L_{\rho^{-1} \xi}^{n} \xi\right|_{\partial M}$ for all $n \in \mathbb{Z}_{+}$. Then all functionals (3.18) will be the generalized helicity invariants of (3.2).

The results obtained above allow some interesting modifications. To present them in detail, observe that equality (3.12) can be rewritten as

$$
\left(\partial / \partial t+L_{u}\right) \beta^{(1)}-d h=\left(\partial / \partial t+L_{u}\right) \tilde{\beta}^{(1)}=0,
$$

where, by definition,

$$
h:=\rho^{-1} P+\frac{1}{2}|u|^{2}, \quad \tilde{\beta}^{(1)}:=\langle u-\nabla \varphi, d x\rangle,
$$

and the scalar function $\varphi: M \longrightarrow \mathbb{R}$ is chosen in such a way that

$$
\left(\partial / \partial t+L_{u}\right) \varphi=\nabla h
$$

Then, obviously, there holds the additional equality

$$
\left(\partial / \partial t+L_{u}\right) d \tilde{\beta}^{(1)}=0
$$

following from the commutation property $\left[d, \partial / \partial t+L_{u}\right]=0$. Then we obtain that the density $\tilde{\mu}:=\tilde{\beta}^{(1)} \wedge d \tilde{\beta}^{(1)} \in \Lambda^{3}(M)$ satisfies the condition

$$
\left(\partial / \partial t+L_{u}\right) \tilde{\mu}=0
$$

for all $t \in \mathbb{R}$. The similar equality holds for densities $\tilde{\mu}_{n}:=L_{v}^{n} \tilde{\mu} \in \Lambda^{3}(M), n \in \mathbb{Z}_{+}$:

$$
\left(\partial / \partial t+L_{u}\right) \tilde{\mu}_{n}=0
$$


owing to the commutation property (2.23). Thereby, the following functionals on the corresponding functional manifold $\mathcal{M}$ are invariants of the superfluid flow (2.26):

$$
\mathfrak{M}_{n}:=\int_{M} \tilde{\mu}_{n}=\int_{D_{t}} \rho L_{\rho^{-1} \xi}^{n}\langle(u-\nabla \varphi), \xi\rangle d^{3} x
$$

for all $n \in \mathbb{Z}_{+}$and an arbitrary domain $D_{t} \subset M$, independent of boundary conditions, imposed on the vorticity vector $\xi=\nabla \times u$ on $\partial M$. Notice here that only invariants (3.25) strongly depend on the function $\varphi: M \longrightarrow \mathbb{R}$, implicitly depending on the velocity vector $u \in T(M)$. Mention here only that the practical importance of the constructed invariants (3.25) is not still clear enough.

\section{Conclusions}

The symplectic and symmetry analysis of compressible MHD superfluid, as shown above, appeared to be effective for constructing the related helicity type conservation laws, important for practical applications. In particular, these conservative quantities play a decisive role [4, 1, when studying the stability of MHD superfluid flows under special boundary conditions.

Here we also need to notice that the differential-geometric reformulation of MHD equations (2.2) suggested in [4] is incorrect. Namely, the equality $\left(\partial / \partial t+L_{u}\right)\left\langle\rho^{-1} B, d x\right\rangle=0$ is not equivalent to the magnetic field equation $\partial B / \partial t-\nabla \times(\nabla \times B)=0$ that one can check by easy calculations. Nonetheless, the commutator relation $\left[\partial / \partial t+L_{u}, L_{\rho^{-1} B}\right]=0$ devised there and all Casimir invariants found in article [4] are true. But some problems related tothe construction of nonCasimir type MHD superfluid flows using their Hamiltonian structure remain, in general, open and wait still to be treated in detail. Some of the results in this direction can be eventually obtained making use of group-theoretical and topological tools developed in [1, 13, 11, where the importance of the basic group of diffeomorphisms $\operatorname{Diff}(M)$ of a manifold $M \subset \mathbb{R}^{3}$ and its differential-geometric characteristics were stated.

\section{Acknowledgments}

Two of the authors (N.B. and A.P.) are cordially thankful to the Abdus Salam International Centre for Theoretical Physics in Trieste, Italy, for the hospitality during their research 2007scholarships. Special thanks are tributed to Prof. J. Slawianowski (IPPT of Warsaw, Poland) for his interest in our research, fruitful discussions and useful comments. Last but not least thanks go to Mrs. Dilys Grilli from the ICTP Publications Office for her very professional help and advice during the preparation of the manuscript. 


\section{References}

[1] Arnold V.I. and Khesin B.A. Topological methods in hydrodynamics. Springer, NY, 1998.

[2] Abraham R. and Marsden J. Foundations of mechanics. Cummings Publ., NY, 1978.

[3] Holm D. and Kupershmidt B. Poisson structures of superfluids. Phys. Lett., 91A (1982), pp. $425-430$.

[4] Holm D., Marsden J., Ratiu T. and Weinstein A. Nonlinear stability of fluid and plasma equilibria. Physics Reports, 123/(1 and 2) (1985), pp. 1-116.

[5] Moffat H.K. The degree of knottedness of tangled vortex lines. Journal of Fluid Mechanics, $35 / 1$ (1969), pp. 117-129.

[6] Owczarek R. Topological defects in superfluid Helium. Int. J. Theor. Phys., 30/12 (1991), pp. $1605-1612$.

[7] Owczarek R. Frames and fermionic excitations of vortices in superfluid Helium. J. Phys: Condens. Matter, 5 (1993), pp. 8793-8798.

[8] Peradzyński Z. Helicity theorem and vertex lines in superfluid ${ }^{4}$ He. Int. J. Theor. Phys., 29/11 (1990), pp. 1277-1284.

[9] Putterman S.J. Superfluid Hydrodynamics, North Holland, Amsterdam, 1974.

[10] Prykarpatsky A. and Mykytiuk I. Algebraic integrability of nonlinear dynamical systems on manifolds: classical and quantum aspects. Kluwer Academic Publishers, the Netherlands, 1998.

[11] Prykarpatsky A. and Zagrodziński J. Dynamical aspects of Josephson type media, Ann. of Inst. H. Poicare, Phys. Theorique, 70/5 (1999), pp. 497-524.

[12] Schwarz K.W. Physical Rev. B, 38 (1988), pp. 2398-2417.

[13] Troshkin O.V. Nontraditional methods in mathematical hydrodynamics. Transl. Math. Monogr., AMS, Providence, v.114, 1995. 